\title{
Conditional protein stabilization via the small molecules Shld-1 and rapamycin increases the signal-to-noise ratio with tet-inducible gene expression
}

\author{
Gal Almogy and Garry P. Nolan \\ BioTechniques 46:44-50 (January 2009) \\ doi 10.2144/000113030
}

\begin{abstract}
Cellular mechanisms control one or more of the three basic levels of regulation (transcription, translation, and protein activity/locality), allowing for finely tuned spatial and temporal regulation of protein expression patterns. Gene regulation constructs in wide use today often employ a constitutively expressed transcription factor whose activity is determined by the presence or absence of a small molecule. A case in point is the tet transcription system, wherein transcription is regulated by doxycycline (Dox), allowing the researcher to turn protein expression on or off, depending on the presence/absence of Dox. However, in many applications of that system, there is basal transcription from the promoter element that is independent of Dox. Moreover, in vivo, heterogeneous distribution of Dox leads to concurrent differences in gene expression. We addressed these limitations by introducing conditional destabilizing elements to the system. First, we created a transactivator protein fusion regulated at the additional level of protein stability. This modification enabled a system that demonstrated an off state that is less sensitive to variations in Dox concentrations. We also regulated the stability of the protein expressed from the tet operator cassette, observing greatly improved signal-to-noise ratios. The results underscore how investigator-defined regulation at multiple levels of protein expression can attain a finer degree of control over the final expression of introduced genes.
\end{abstract}

\section{INTRODUCTION}

The ability to introduce and express exogenous DNA in a controlled manner in mammalian cells plays an important role in the study of biological phenomena as well as in enabling the generation of new tools for therapeutic ends, such as gene therapy $(1,2)$. Many approaches have been developed to exactly control the spatial and temporal expression of exogenous genes, typically applying the regulation at the transcriptional level and thereby controlling messenger RNA (mRNA) copy number and subsequently protein level (2), but other, more complex regulation systems have been implemented (3-7).

One of the best transcriptional regulatory systems, often termed the tet inducible system, consists of two components, the tetracycline transcription activator protein (tTA) and a DNA operator sequence, tet $O(8-10)$. The transactivator is a chimeric protein consisting of the VP16 transcription activation domain and the Escherichia coli tet-repressor DNA binding domain. In the Tet-Off version, in the absence of the tetracycline derivative doxycycline (Dox), the tet repressor binds to the tet $O$ sequence, bringing the VP16 subunit close enough to DNA to initiate transcription of the regulated gene. Dox addition induces a conformational change in the tet repressor, which prevents the association of the tet repressor with the DNA; thus, when Dox is added, transcriptional activity (mediated by VP16) is repressed. The Tet-Off system has been used extensively in vitro and in vivo for protein expression purposes, yet some shortcomings still exist that limit its utility: most notably, detectable background expression occurs even in the continual presence of Dox. Furthermore, the system is not protected from Dox fluctuations in the sense that a small decrease in the concentration of Dox would render the system active, the extent of activity depending on the concentration of Dox.

Another synthetic regulatory system is based on the ability to control protein stability $(11,12)$ via small molecule intervention. FKBP12-rapamycin binding domain of mTOR (FRB) is a highly unstable 89-amino acid destruction domain derived from the mTOR protein $(13,14)$ that is rapidly degraded in cells. When expressed as a fusion with another protein, this instability is conferred on the fused protein in a cis-dominant manner, leading to rapid turnover of the fusion protein in mammalian cells. Rapamycin (Rap) is a natural antifungal, cell-permeable compound also capable of serving as an immunosuppressant by inhibiting the activity of the mTOR pathway $(13,14)$. Notably, FRB and FRB-fusion proteins are stabilized when Rap is added to the cellular media (15), but as Rap is an mTOR pathway inhibitor, its use in cellular systems may be challenging. In response to this potential complication, other inducibly stabilized domains have been developed that are stabilized by what so far have been shown to be substances that do not affect cellular activity $(15,16)$. For instance, FRB variants (i.e., FRB*; see Reference 15) can respond to a rapamycin substitute, and the FKBP* inducibly stabilized 


\section{Research Reports}

domain is responsive to the biologically inert synthetic molecule Shld-1 (16).

We investigated how regulating protein stability may be introduced into the tTA system and define two modes of combining regulatory modules (17). In the first, the parallel regulation approach, destabilization is applied to the control protein (tTA). In the second, the serial regulation approach, the destabilization is applied to the protein already regulated on the transcriptional level. We show that each strategy has very distinct advantages and applications.

\section{MATERIALS AND METHODS}

\section{Vectors}

A sequence coding for transcription initiation followed by the FRB* coding sequence was cloned into the MFG-I-CD8 vector, a constitutive retroviral expression vector for expressing a gene of interest followed by an internal ribosomal entry site (IRES) element, and CD8 as a marker. The resulting construct, MFG-FRB*, contains $\mathrm{FRB}^{*}$ inserted within the MluI and SalI restriction sites. We used primers for the $\mathrm{N}$ and $\mathrm{C}$ termini of tTA in the Retrotet RTAb(-) vector to PCR-amplify a tTA coding sequence containing no start codon. This tTA (lacking the first ATG) cDNA was then cloned in-frame into MFG-FRB* using SalI and NotI restriction sites. The resultant vector expresses an FRB*-tTA fusion protein (termed $\mathrm{F}^{*} \mathrm{tTA}$ hereafter) and an IRES-expressed cell surface marker, CD8. The same procedure was carried out to produce $\mathrm{K} * \mathrm{tTA}$, which is a fusion of FKBP* and tTA. Unless mentioned otherwise, the fusion proteins are separated only by the two amino acids coded by the SalI site (GTCGAC). The MFG-I-CD8 and Retrotet vectors were gifts from Helen Blau (Stanford
University, Palo Alto, CA, USA), and FKBP* cDNA was provided by Tom Wandless (Stanford University).

The reporter vector HRG was created by cutting the IRES-green fluorescent protein (GFP) sequence from the HRI-GFP vector (gift from Dr. Helen Blau) using XhoI and NotI restriction enzymes and inserting the enhanced GFP (EGFP) cDNA in its place. The resultant vector contains seven repeats of the tet operator sequence followed by the EGFP coding sequence. The $\mathrm{HRK}^{*} \mathrm{G}$ reporter vector is similar to $H R G$, but instead of EGFP, it contains an FKBP*EGFP fusion (K*EGFP). The $\mathrm{K}^{*} \mathrm{EGFP}$ fragment was obtained from the $\mathrm{MK}^{*} \mathrm{G}$ vector, which was itself constructed by introducing an FKBP*EGFP fusion into the previously described MFG-I-CD8 retroviral backbone in a process analogous to that used to create $\mathrm{K} * \mathrm{tTA}$.

\section{Virus Production}

293T cells were transfected using the modified MFG/HRG retroviral vectors and the pCL-eco packaging vector (Invitrogen, Carlsbad, CA, USA) using Fugene6 transfection reagent (Roche, Indianapolis, IN, USA) in $10 \%$ fetal calf serum (FCS; Invitrogen) media in a $10-\mathrm{cm}$ plate. Media was replaced with $10 \mathrm{~mL}$ fresh media $24 \mathrm{~h}$ posttransfection, and virus particles were collected $48 \mathrm{~h}$ posttransfection and used immediately (no purification).

\section{Cells}

NIH/3T3 (murine) served as the basic testing cell line. Cells were transduced with the various transactivator constructs (i.e., tTA, F*tTA) using retroviral particles (described above in "Virus

\section{Redefining the cell-based assay!}

\section{5 years of experience with impedance-based cell assays put into one instrument line!}

Measurements are rapid, accurate and highly reproducible. ECIS monitors cell behavior in tissue culture quantitatively and in real time. The ECIS Z \& Z $\theta$ are the new standard in life sciences for impedance-based quantification of cell behavior in vitro.

To learn more or to demo ECIS visit www.biophysics.com or call 1.866.301.ECIS (3247).

\section{(ab) Apolled BioPhysics} Quantifying Cell Behavior
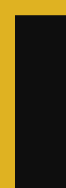


\section{Research Reports}

Production") in the presence of $1 \mu \mathrm{g} / \mathrm{mL}$ polybrene (Sigma, St. Louis, MO, USA). Cells expressing the various transactivators were sorted using anti-CD8 PE-conjugated antibodies (giving 95\% purity of CD8-expressing cells). Unless otherwise mentioned, 3T3 cells sorted for CD8 expression were then transduced with the different reporter constructs ( $1 \mathrm{~mL}$ of viral supernatant per 50,000 cells). Thirty-six hours after transduction,
$1 \mathrm{nM}$ stabilizer (Rap/Shld-1) was added. EGFP-positive cells were sorted after 24 $\mathrm{h}$ using a FacsCalibur machine (Becton Dickinson and Company, Franklin Lakes, NJ, USA). Sorted cells were incubated in standard media containing Dox (no stabilizer, unless otherwise specified). Cells were grown in Dulbecco's modified Eagle medium (DMEM; Gibco, Invitrogen) containing 10\% FCS and penicillin-streptomycin-l-glutamine (PSQ).
A
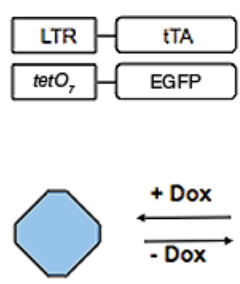

$$
\text { a. ITA }
$$

inactive

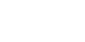

(TTR
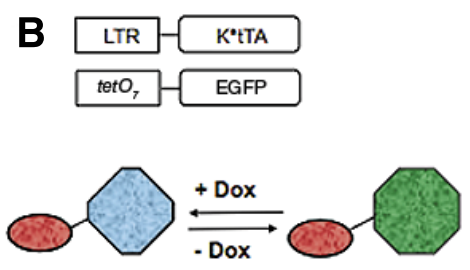

c. K*tTA

inactive, unstable d. K*tTA

active, unstable
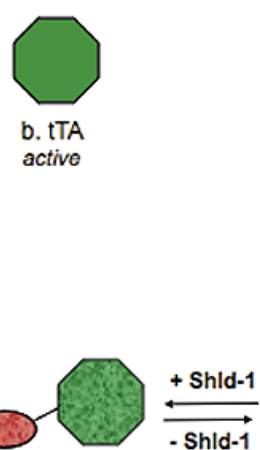

-Shld-1

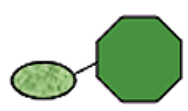

e. K*tTA active, stable
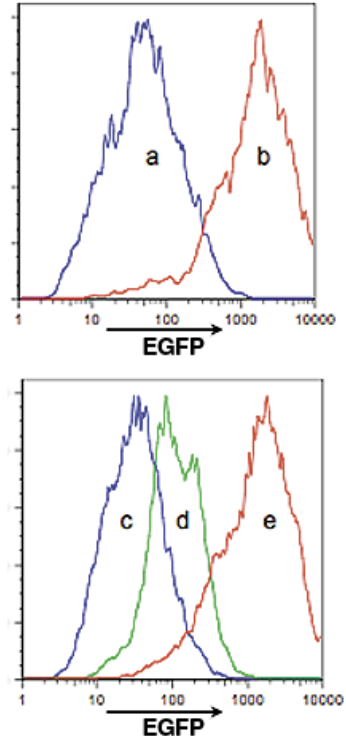

\section{Induction/Suppression}

Cells were cultured in $50 \mathrm{ng} / \mathrm{mL}$ Dox-containing media and induced by replacing with Dox-free media (Invitrogen) for $24 \mathrm{~h}$. Cells carrying the conditionally destabilized transactivators/ reporter proteins were induced by adding the stabilizer $1 \mathrm{nM}$ Rap (Sigma) or $1 \mu \mathrm{M}$ Shld-1 (gift from Tom Wandless) in the absence of Dox. The typical induction time until full signal was reached was $24 \mathrm{~h}$. To suppress EGFP, expression cells were cultured in $50 \mathrm{ng} / \mathrm{mL}$ Dox-containing media (in the absence of stabilizer); media was replaced every $24-48 \mathrm{~h}$ with fresh Dox-containing media. Cells were incubated for $>7$ days in Dox-containing media before every induction experiment to ensure suppression.

\section{Assay and Analysis}

Cells were detached using Trypsin plus $0.5 \mathrm{mM}$ EDTA, washed in phosphatebuffered saline (PBS), and then analyzed for EGFP expression using a FacsCalibur flow cytometer. Quantitative results, unless otherwise stated, are presented as the averaged value of three independent measurements.

Figure 1. Flow cytometry analysis of transactivator activity of classic tetracycline transcription activator protein (tTA) and conditionally stabilized tTA constructs. (A) The vectors used in this experiment are depicted in the upper left-hand corner of the panel, wherein a retroviral $5^{\prime}$ long terminal repeat (LTR) constitutively expresses the tTA protein, and green fluorescent protein (GFP) is driven by a promoter containing seven tet operator sequences $\left(\right.$ tet $\left._{7}\right)$. In the absence of doxycycline (Dox), the active tTA protein binds to the tet operator DNA sequence and initiates transcription, leading to high expression of enhanced GFP (EGFP) (red histogram in the flow cytometry plot). Addition of Dox induces a conformational change in the tTA, rendering it incapable to bind DNA and thereby inhibiting transcription (blue histogram). (B) The vectors used in this experiment are depicted in the upper left-hand corner of the panel, wherein a retroviral 5' LTR constitutively expresses the K*tTA protein, and GFP is driven by a promoter containing seven tet operator sequences $\left(\operatorname{tet}_{7}\right)$. In the absence of Dox/Shld-1, the $\mathrm{K}^{*} \mathrm{tTA}$ protein is active but unstable, leading to low-level transcriptional activity (green histogram). Addition of Shld-1 in the absence of Dox stabilizes the K*tTA and initiates full transcriptional activity (red histogram). Addition of Dox in the absence of Shld- 1 induces a conformational change in the tTA, rendering it incapable to bind DNA and thereby inhibiting transcription (blue histogram). x-axis, fluorescence (arbitrary units); y-axis, percentage.

Table 1. Basic Comparison of the Various Conditionally Destabilized Transactivators Activity Profile

\begin{tabular}{|c|c|c|c|c|c|c|}
\hline Stabilizer & - & - & + & + & - & \\
\hline $\begin{array}{l}\text { Activator Construct/ } \\
\text { Reporter }\end{array}$ & \multicolumn{5}{|c|}{ Median Fluorescence Intensity (MFI) } & $\begin{array}{l}\text { Inducibility } \\
\text { Factor }\end{array}$ \\
\hline $\begin{array}{l}\text { LTR-tTA } \\
\text { tetO }_{7} \text {-EGFP }\end{array}$ & $51 \pm 7$ & $2800 \pm 140$ & - & - & $1101 \pm 270$ & 62 \\
\hline $\begin{array}{l}\text { LTR-F*tTA } \\
\text { tetO }_{7} \text {-EGFP }\end{array}$ & $29 \pm 6$ & $95 \pm 9$ & $35 \pm 2$ & $740 \pm 73$ & $49 \pm 23$ & 32 \\
\hline $\begin{array}{l}\text { LTR-K*tTA } \\
\text { tetO } O_{7} \text {-EGFP }\end{array}$ & $38 \pm 5$ & $154 \pm 15$ & $44 \pm 4$ & $2300 \pm 131$ & $73 \pm 19$ & 72 \\
\hline $\begin{array}{l}\text { LTR-tTA } \\
\text { tet } O_{7}-K^{\star} \text { EGFP }\end{array}$ & $6.7 \pm 1$ & $215 \pm 23$ & $46 \pm 3$ & $1850 \pm 52$ & $149 \pm 35$ & 2600 \\
\hline
\end{tabular}




\section{Research Reports}

\section{RESULTS}

\section{Control Module Engineered to Regulate the Stability of a Transcriptional Activator}

The modified tet-inducible system developed in this section is composed of two vectors: $(i)$ a regulator vector constitutively expressing the classic tTA or a stability-modulated tTA from a viral $5^{\prime}$ long terminal repeat (LTR) promoter and (ii) a classic reporter vector (termed here $H R G$ ) carrying seven repeats of the tet operator $\left(t^{2 t} \mathrm{O}_{7}\right)$ followed by EGFP cDNA (18). For stability-modulated tTA, we combined the tTA reading frame with cDNA encoding either FRB* (15) or FKBP* (16) to create the F*tTA and $\mathrm{K} * \mathrm{tTA}$ conditionally destabilized transactivator fusion proteins, respectively.

While the standard tTA system in 3T3 cells carrying the reporter vector HRG has ON and OFF activation states (Figure 1A, red and blue flow cytometry histograms, respectively) provided by the absence and presence of Dox, the destabilized tTA system (Figure 1B) should have an adjunct regulatory state brought about via conditional destabilization of the tTA protein. To test this, we created other cell lines constitutively expressing either the $\mathrm{F}^{*} \mathrm{tTA}$ or $\mathrm{K} * \mathrm{tTA}$ protein and carrying the reporter vector HRG. As shown with results for $\mathrm{K}^{*} \mathrm{tTA}$, the resulting cells have an ON state (Figure 1B, red line), an OFF state (Figure 1B, blue line), and a state of partial activation (Figure 1B, green) in the absence of both Dox and stabilizer.

As summarized in Table 1, addition of a tTA fusion stabilizer (Rap for F*tTA or Shld-1 for $\mathrm{K}^{*} \mathrm{tTA}$ ) in the absence of Dox (ON condition) gives rise to robust EGFP expression (+Stabilizer/-Dox column), whereas addition of Dox in the absence of stabilizer (OFF condition) leads to inhibition of transcriptional activity. Although the $\mathrm{F}^{*} \mathrm{tTA}$ - and $\mathrm{K} * \mathrm{tTA}$-based systems appear to have slightly lower background activity levels than tTA, the -Stabilizer/+Dox condition does not eliminate EGFP expression driven by the $\mathrm{F}^{*} \mathrm{tTA}$ or $\mathrm{K}^{*} \mathrm{tTA}$ regulators. In the absence of both drugs (-Stabilizer/-Dox), the $\mathrm{F}^{*} \mathrm{tTA}$ and $\mathrm{K} * \mathrm{tTA}$ systems have an additional, distinct control state, where they exhibit partial activation. Under these partial activation conditions, there are drastically reduced levels of the destabilized transactivator; however, since Dox is not present, the remaining transactivator fusion proteins retain activity, leading to low-level transcription. In the presence of both drugs (+Stabilizer/+Dox), the transcription-activating fusion protein is stabilized, but its transcriptional activity is repressed, leading to slightly elevated EGFP expression compared with the OFF state [median fluorescence intensity (MFI) of 35 versus 29 for $\mathrm{F}^{*} \mathrm{tTA}$, and 44 versus 38 for $\mathrm{K} * \mathrm{tTA}$ ], but not elevated enough to consider it a useful independent activation state.

In the case of the $\mathrm{K}^{*}$-tTA fusion, we observed slightly improved inducibility, defined as the ratio of the normalized maximal expression level (expressed as MFI) for the ON condition over the normalized MFI for the OFF condition:

$$
\frac{\mathrm{MFI}_{\mathrm{ON}}-\mathrm{MFI}_{\text {background }}}{\mathrm{MFI}_{\mathrm{OFF}}-\mathrm{MFI}_{\text {background }}} .
$$

\section{Increased Signal-to-Noise Ratio with Conditional Destabilization of a Transcriptionally Regulated Protein}

We next explored whether an addi[Eq. 1] tional level of control could be

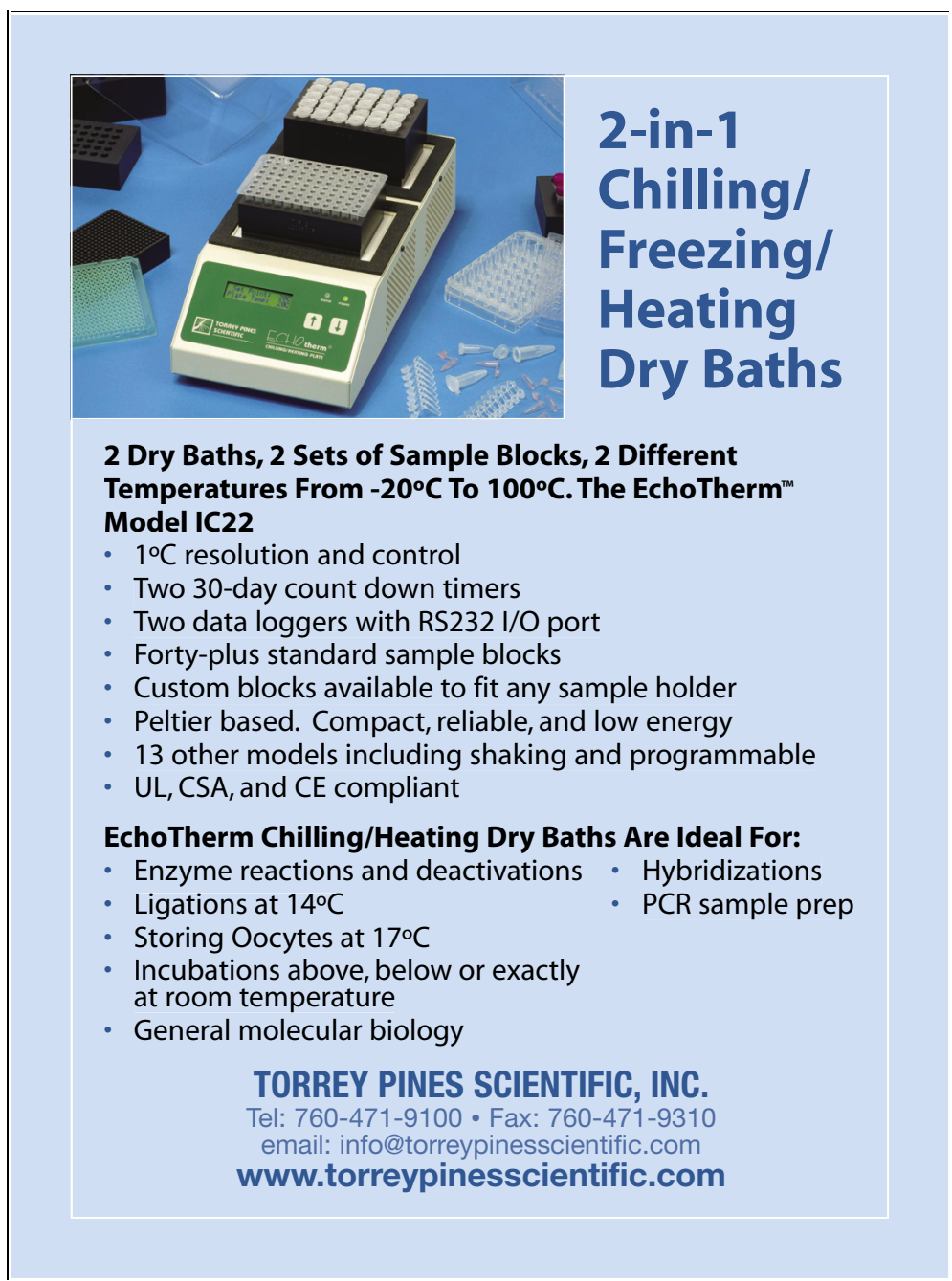




\section{Research Reports}

A

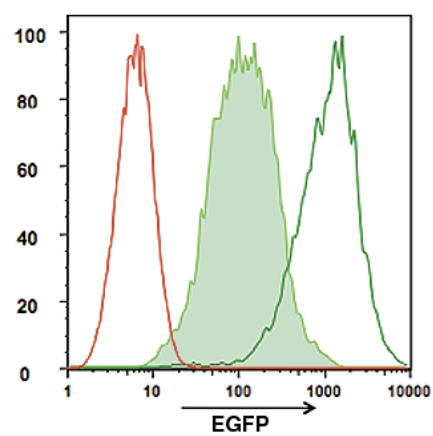

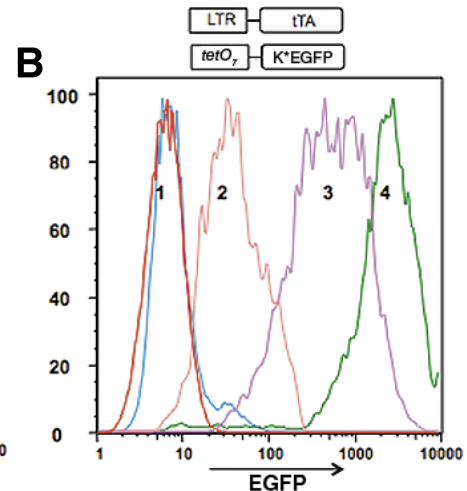

Figure 2. Regulation of enhanced green fluorescent protein (EGFP) levels using reporter protein destabilization. (A) $\mathrm{K}^{*} \mathrm{EGFP}$ is constitutively expressed from the long terminal repeat (LTR) promoter, but the protein product is unstable [light green, filled histogram in the flow cytometry plot; median fluorescence intensity (MFI) of 140]. Addition of stabilizer (Shld-1) results in an increase in expression level by 1 order of magnitude (dark green histogram, MFI of 1350). Naive $3 \mathrm{~T} 3$ cell background fluorescence is shown for comparison (red histogram). (B) $\mathrm{K} *$ EGFP expression is controlled by tetracycline transcription activator protein (tTA) via the $t^{2} t O_{7}$ promoter. Addition of doxycycline (Dox) shuts down tTA activity and subsequent transcription from the inducible promoter, inhibiting the production of $\mathrm{K}^{*} \mathrm{EGFP}$ [-Stabilizer/+Dox, peak 1 (blue histogram), MFI of 6]. Addition of Shld-1 stabilizes K*EGFP, leading to a modest increase in signal (+Stabilizer/+Dox, peak 2, MFI of 40). Removal of Dox induces tTA activation; in the absence of Shld-1, K*EGFP is unstable, leading to another marginal increase in signal (-Stabilizer/-Dox, peak 3, MFI of 220). Addition of Shld-1 stabilizes K*EGFP and induces maximal signal (+Stabilizer/-Dox, peak 4, MFI of 1870).

achieved by using a conditionally destabilized reporter. First, to verify the previously published inducibility levels for protein stabilization $(15,16)$, we transduced naive $3 \mathrm{~T} 3$ cells with $\mathrm{MK} * \mathrm{G}$, a vector constitutively expressing the $\mathrm{K}^{*}$ EGFP fusion protein, and then cultured these cells in the absence (Figure 2A, light green, filled histogram) and presence (Figure 2A, dark green line) of the stabilizing drug Shld-1. This conditional destabilization/stabilization of the reporter protein led to an induction ratio of 10 , which is similar to published reports (16). Compared with naive 3T3 cells (Figure 2A, red line), constitutive baseline expression level (MFI of 180) was significantly higher than background and about three times greater than that observed with tTA regulation of the HRG reporter vector (MFI of 51; see Table 1).

We then tested the $\mathrm{K}^{*}$ EGFP reporter fusion in the context of an inducible transcription system. For these experiments, we used cells containing tTA and the $\mathrm{HRK}^{*} \mathrm{G}$ reporter vector, which contains seven copies of the tet operator sequence followed by the $\mathrm{K} * \mathrm{EGFP}$ coding sequence. Note that there are two independent control mechanisms in this system: (i) Dox serves to shut down transcription through tTA and (ii) the absence of Shld-1 destabilizes the expressed reporter protein. Thus, the only true OFF condition for the composite system is -Stabilizer/+Dox, where EGFP expression is inhibited at both levels of regulation. Background expression in the OFF state was almost identical to the 3T3 background fluorescence [Figure 2B, compare background fluorescence (red line) and peak 1 (blue line)]. Notably, despite extremely low background levels of EGFP fluorescence, the composite system maintained a potent signal in the ON state (+Stabilizer/-Dox, peak 4). As a result, the composite system showed $>1$ order of magnitude more inducibility than when EGFP (unfused to FKBP*) was regulated by either tTA or K*tTA (the inducibility factor of LTR-tTA/ tet $O_{7}-\mathrm{K}^{*} \mathrm{EGFP}$ is 2600 , compared with 72 for LTR-K*tTA/tet $O_{7}$-EGFP, a 36-fold increase; see Table 1).

Moreover, unlike cells transduced with the tTA and the standard EGFP reporter HRG, cells expressing tTA and $\mathrm{K}^{*}$ EGFP display two distinct stable expression states beyond a simple ON/OFF, as seen in Figure 2B. In the presence of both drugs (+Stabilizer/+Dox) the transcriptional system is off, but the reporter protein is stabilized, leading to a significant increase in signal that is distinct from that observed in the OFF state (Figure $2 \mathrm{~B}$, peak 2). The composite system also has an additional control state in the absence of both drugs (-Stabilizer/-Dox), where transcription is active but the protein product is unstable (Figure 2B, peak 3 ). Note that in this system-as in the $\mathrm{F}^{*} \mathrm{tTA}$ (or $\mathrm{K}^{*} \mathrm{tTA}$ ) system described above-both Dox and the stabilizing drug (i.e., Shld-1) maintained their independent qualities, that is, both drugs were still fully titratable as they were with the independent components by themselves. Supplementary Figure 1 (available at www.BioTechniques.com) depicts a Western blot analysis of Shld-1 titration in the absence of Dox, demonstrating that Shld-1 addition is titratable and that the EGFP signal as measured by flow cytometry correlates with EGFP protein levels.

Hypothetically, EGFP signal should decay more rapidly with the destabilizing fusion domain than in the more classic system, as the protein product has a high turnover rate in the absence of stabilizer. To test this hypothesis, we created tTA/ EGFP and tTA/K*EGFP cell lines and compared the EGFP signal decay kinetics between the two, by first applying the ON conditions (+Stabilizer/-Dox) and, after $24 \mathrm{~h}$, switching back to the OFF conditions (-Stabilizer/+Dox). There was little difference in induction time between the two cell lines, with both reaching maximal EGFP levels within $24 \mathrm{~h}$ of induction (Figure 3 ).

However, $48 \mathrm{~h}$ after the OFF state was initiated, tTA/EGFP cells still showed a positive phenotype with an MFI of 880 (compared with maximal MFI of 2300), whereas the tTA/K*EGFP cells showed an MFI of 11 (compared with 1800 maximal MFI) — essentially returning to the baseline MFI of 7. After an additional $72 \mathrm{~h}(5 \mathrm{~d}$ after the addition of Dox) the tTA/EGFP population finally showed an almost complete return to the suppressed (OFF) phenotype with an MFI of 63 (compared with a baseline of 52 ), while the tTA/K*EGFP population was completely suppressed. Thus, the composite system, where expression is serially regulated via both transcription and reporter stability, demonstrates more complete and finer temporal control overexpression.

\section{DISCUSSION}

We investigated two methods of regulatory control $(6,17)$ in which the stability of the transcriptional activator or the final protein produced is regulated externally via small molecule intervention. In the first approach, a conditionally destabilized tTA fusion (i.e., $\mathrm{K} * \mathrm{tTA}$ ) was developed to create a transactivator that was controlled in a parallel fashion by two different drugs: Dox for 

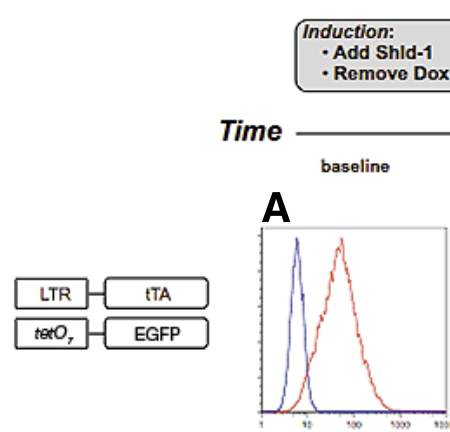

E
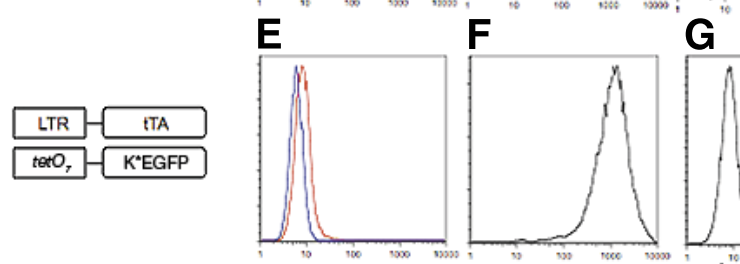
19000009
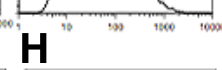

D 120 hours postrot
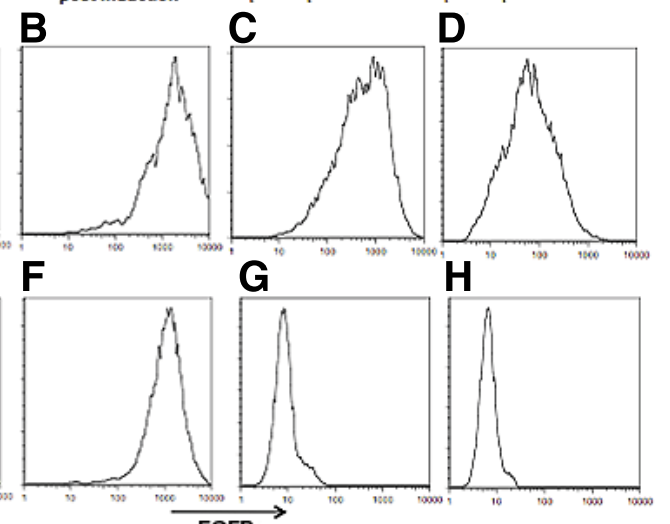

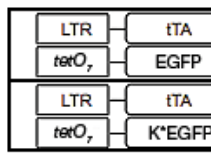

$52(103)$

\begin{tabular}{|c|c|c|}
\hline $2300(2081)$ & $880(936)$ & $63(109)$ \\
\hline $1835(944)$ & $10.8(12)$ & $7(8.1)$ \\
\hline
\end{tabular}

MFI (standard deviation)

Figure 3. Fluorescence-activated cell sorting (FACS) analysis of HRK*G vector suppression kinetics compared with HRG vector. The upper panel shows an unmodified enhanced green fluorescent protein (EGFP) as the reporter gene, while the lower panel shows results using K*EGFP. Initially, the system is at resting condition (-Stabilizer/+Dox) and shows baseline expression (A and E); median fluorescence intensities (MFIs) are tabulated at the base of the figure and are 52 and 6, respectively (standard deviations are shown in parentheses). B and F show maximal expression levels (2300 and 1850 , respectively) after removal of doxycycline (Dox) and addition of Shld-1. Twenty-four hours post-stimulus, we removed Shld-1 and added Dox to initiate the OFF signal. Forty-eight hours after the OFF signal, LTR-tTA/tet $O_{7}$-EGFP cells (C) showed a slightly reduced EGFP-positive phenotype (MFI of 880, versus 2300 at maximal induction), and the signal was reduced to prestimulation levels only $120 \mathrm{~h}$ after the OFF signal (D). In contrast, $48 \mathrm{~h}$ after OFF signal, the LTR-tTA/tet $O_{7}-\mathrm{K} *$ EGFP population $(\mathrm{G})$ showed an already far-reduced EGFP signal (11 versus 1835 at maximum), and $120 \mathrm{~h}$ post-OFF signal, the entire population returned to the OFF state $(\mathrm{H})$. LTR, long terminal repeat; tTA, tetracycline transcription activator protein.

transcriptional regulation and Shld-1 for control of protein stability.

The conditionally destabilized tTA versions we studied consistently showed less transcriptional activity from the reporter vector when in the repressed state $(P<0.01$, Student's $t$-test $)$. These reduced basal expression levels may be due to less-than-complete repression of unmodified tTA in the presence of saturating amounts of Dox, and/or the short time the cells spend in Dox-free media when cells are washed during culture (9). In addition, conditionally destabilized tTA has a limited range of activation in the absence of a stabilizer, offering a potential advantage in vivo, where Dox concentration may vary. As shown in Table 1, the conditionally destabilized $\mathrm{K}^{*} \mathrm{tTA}$ shows only very limited activity in the absence of Shld-1 [MFI of 150 (-Stabilizer/-Dox) versus MFI of 2300 (+Stabilizer/-Dox)]. Moreover, while a reduction in Dox concentration (1/50 of maximal concentration used) leads to very significant activation of the standard tTA system (leading to $40 \%$ of maximal MFI), the same conditions barely have any effect on the destabilized tTA $\left(\mathrm{F}^{*} / \mathrm{K}^{*} \mathrm{tTA}\right)$, with $\mathrm{MFI}$ reaching $-3 \%$ of maximal, as shown in Table 1 .

Layering transcription control over a destabilized protein in a serial fashion (i.e., Dox-regulated transcription of the transactivator and stabilizer-controlled stability of the reporter) provided a significant improvement in the signalto-noise ratio, four distinct activation states, and a tighter temporal window of induction, implying that the expression of a gene of interest (i.e., EGFP) may be better regulated using an inducible destabilization scheme in which the target of inducible destabilization is the final expressed protein product. Furthermore, by using the serial control scheme, one can theoretically control transcriptional fluctuations in a manner that is independent from complicating factors, such as chromosomal integration site effects, commonly observed with retroviral inserts. Interestingly, the serial approach does not protect the system from fluctuations in Dox concentration nearly as much as the parallel approach (see Table 1, where the signal is $>22$
Research Reports

times higher than background when the Dox concentration is $1 / 50$ the normal concentration).

There are some technical drawbacks to the overall approach. For instance, it would not be expected that all proteins are stabilized/destabilized to the same extent by fusion to the domains used in this report (note that this limitation is not relevant to the parallel regulation approach). In addition, some proteins, when fused to the destabilizing domains, might be rendered inactive simply due to the fusion event itself. As such, the approach might not be useful for all protein tests. For example, multipass transmembrane proteins are not yet amenable to this technique (16), and other proteins may not be significantly affected by these particular fusions (i.e., Cre recombinase, data not shown). Thus, researchers must verify that their protein of interest is indeed destabilized by the fusion and that the function of the protein itself is not altered. Additionally, the Rapamycin (FK506) used in these experiments affects endogenous cellular signaling processes and is thus not suitable for all studies or applications, especially those in vivo. However, both FRB* and FKBP* may be stabilized by biologically inactive substances $(15,16)$ and thus may possibly be used in human contexts (i.e., Shld-1). In conclusion, these vector systems provide new regulatory modules for improved quantitatively and temporally exact gene expression in basic research and for potential gene therapy applications in vivo.

\section{ACKNOWLEDGMENTS}

The authors thank the Nolan laboratory, particularly Angelica Trejo and Howard Guss, for their contributions to this work. This work was supported by the National Institutes of Health (grant nos. NIH U54 CA119367 and NIH P01 CA34233), and the Leukemia and Lymphoma Society (grant no. LLS 701706). This paper is subject to the NIH Public Access Policy.

\section{COMPETING INTERESTS STATEMENT}

The authors declare no competing interests. 


\section{Research Reports}

\section{REFERENCES}

1. Zoltick, P.W. and J.M. Wilson. 2001. Regulated gene expression in gene therapy. Ann. N.Y. Acad. Sci. 953:53-63.

2. Heine, H.L., H.S. Leong, F.M. Rossi, B.M. McManus, and T.J. Podor. 2005. Strategies of conditional gene expression in myocardium: an overview. Methods Mol. Med. 112:109-154.

3. Malphettes, L. and M. Fussenegger. 2006. Improved transgene expression fine-tuning in mammalian cells using a novel transcriptiontranslation network. J. Biotechnol. 124:732-746.

4. Malphettes, L., R.G. Schoenmakers, and M. Fussenegger. 2006. 6-hydroxy-nicotine-inducible multilevel transgene control in mammalian cells. Metab. Eng. 8:543-553.

5. Rossi, F.M., A.M. Kringstein, A. Spicher, O.M. Guicherit, and H.M. Blau. 2000. Transcriptional control: rheostat converted to on/off switch. Mol. Cell 6:723-728.

6. Sudomoina, M., E. Latypova, O.O. Favorova, E.A. Golemis, and I.G. Serebriiskii. 2004. A gene expression system offering multiple levels of regulation: the Dual Drug Control (DDC) system. BMC Biotechnol. 4:9.

7. Zhao, H.F., J. Boyd, N. Jolicoeur, and S.H. Shen. 2003. A coumermycin/novobiocin-regulated gene expression system. Hum. Gene Ther. 14:1619-1629.

8. Gossen, M., A.L. Bonin, and H. Bujard. 1993. Control of gene activity in higher eukaryotic cells by prokaryotic regulatory elements. Trends Biochem. Sci. 18:471-475.

9. Gossen, M. and H. Bujard. 1992. Tight control of gene expression in mammalian cells by tetracycline-responsive promoters. Proc. Natl. Acad. Sci. USA 89:5547-5551.

10. Gossen, M. and H. Bujard. 2002. Studying gene function in eukaryotes by conditional gene inactivation. Annu. Rev. Genet. 36:153-173.

11. Roos-Mattjus, P. and L. Sistonen. 2004. The ubiquitin-proteasome pathway. Ann. Med. 36:285-295.

12. Auld, K.L. and P.A. Silver. 2006. Transcriptional regulation by the proteasome as a mechanism for cellular protein homeostasis. Cell cycle 5:15031505 .

13. Stankunas, K., J.H. Bayle, J.E. Gestwicki, Y.M. Lin, T.J. Wandless, and G.R. Crabtree. 2003. Conditional protein alleles using knockin mice and a chemical inducer of dimerization. Mol. Cell 12:1615-1624.

14. Rohde, J., J. Heitman, and M.E. Cardenas. 2001. The TOR kinases link nutrient sensing to cell growth. J. Biol. Chem. 276:9583-9586.

15. Bayle, J.H., J.S. Grimley, K. Stankunas, J.E. Gestwicki, T.J. Wandless, and G.R. Crabtree. 2006. Rapamycin analogs with differential binding specificity permit orthogonal control of protein activity. Chem. Biol. 13:99-107.

16. Banaszynski, L.A., L.C. Chen, L.A. MaynardSmith, A.G. Ooi, and T.J. Wandless. 2006. A rapid, reversible, and tunable method to regulate protein function in living cells using synthetic small molecules. Cell 126:995-1004.

17. Almogy, G., L. Stone, and N. Ben-Tal. 2001. Multi-stage regulation, a key to reliable adaptive biochemical pathways. Biophys. J. 81:30163028.

18. Rossi, F.M., O.M. Guicherit, A. Spicher, A.M. Kringstein, K. Fatyol, B.T. Blakely, and H.M. Blau. 1998. Tetracycline-regulatable factors with distinct dimerization domains allow reversible growth inhibition by p16. Nat. Genet. 20:389393.

Received 21 November 2007; accepted 16 October 2008.

Address correspondence to Garry $P$. Nolan, the Baxter Laboratory in Genetic Pharmacology, Stanford University School of Medicine, Palo Alto, CA, 94305, USA. e-mail: gnolan@stanford.edu

To purchase reprints of this article, contact: Reprints@BioTechniques.com

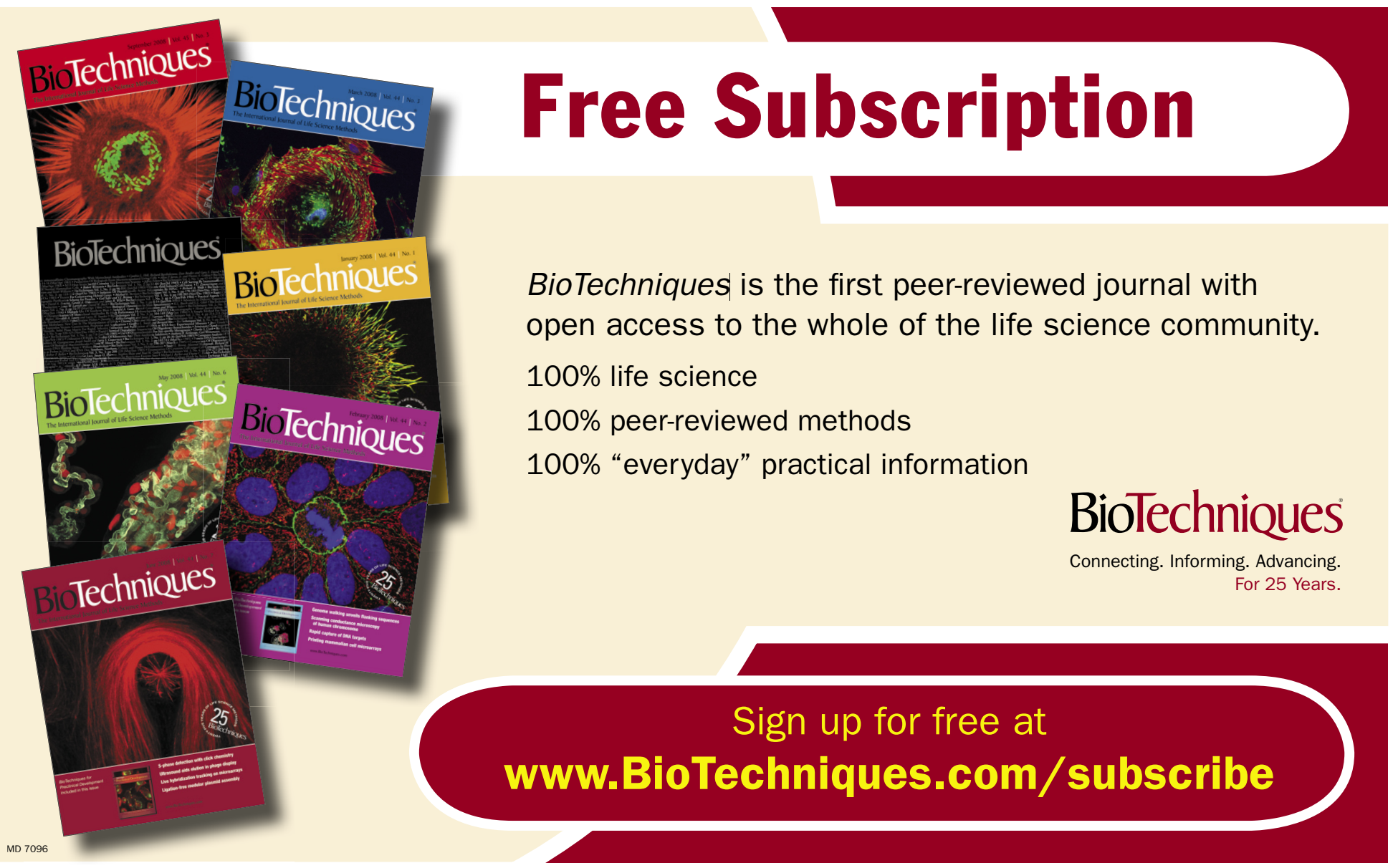

